\title{
Energy-filtered Diffracted Beam Interferometry/Holography (DBI/H)
}

\author{
R.A. Herring ${ }^{*}$ and T. Tanji** \\ ${ }^{*}$ CAMTEC, Mechanical Engineering, University of Victoria, Canada V8W 3P6 \\ ** EcoTopia Science Institute, Nagoya University, Japan 461-8603
}

Energy-filtered $\mathrm{DBI} / \mathrm{H}[\mathbf{1}, \mathbf{2}, \mathbf{3}]$ is a method of interference that can be used to measure on the diffraction plane the intensity and coherence (degree of coherence $(\gamma)$ and lateral spatial coherence $(\delta)$ ) of elastically and inelastically scattered electrons from any two beams or regions of diffraction space by means of an electron biprism. An imaging energy filter (e.g., GIF) is used to separate the zero loss electrons and phonon loss electrons from the plasmon loss electrons, ionization edge loss electrons, etc. A condenser aperture focused on the diffraction plane is used to separate out the zero loss electrons from the phonon loss electrons using their intensity difference due to scattering where the phonon loss electrons dominate the intensity at high angles from the optic axis [3]. The information gained from holograms, as shown in Fig. 1, give a better understanding of the role of energy loss electrons to the contrast of fringes produced in lattice images, which has resulted in a relationship describing the Stobbs factor (SF) expressed as,

$$
\mathrm{SF} \sim \frac{\beta}{\gamma \exp \left(-\pi^{2}|\delta \mathbf{k}|^{2} R_{s}^{2}\right)}
$$

where $\delta \mathbf{k}$ is the beam separation from the exact overlay position, $R_{\mathrm{S}}$ is the effective source size, and $\beta$ is the beam coherence assumed in the simulation usually taken as 1 . Missing in Equ 1 is the specimen thickness, composition, Cs and defocus that would be taken into account by the beam amplitudes. Equ. 1's verification requires taking a lattice image under carefully controlled and measured experimental conditions and then comparing the coherence measured by $\mathrm{DBI} / \mathrm{H}$ for all contributions of intensity. Some confidence that these experiments are proceeding in the right direction is provided by the significant difference between the coherence of the electrons on the optic axis as compared to the high scattering angles. Unexplained is the low coherence of the diffracted beams, which are typically $<0.5$ and found to increase with newer, more stable microscopes. Experiments are now being conducted to test whether chromatic aberration is responsible for the low coherence as the diffracted beams travel a longer path length to the observation plane than the 000 beam.

If the electron experiences an energy loss event inside the crystal specimen by the formation of a quasiparticle such as a plasmon or phonon, the effective source, i.e., $R_{\mathrm{s}}$ in Equ. 1, then becomes the lateral spatial extension of the quasiparticle at the place of the event. Using energyfiltered $\mathrm{DBI} / \mathrm{H}$, for the first time, $\delta$ has been measured for the phonon. Using a Germanium crystal, the phonon loss electrons had $\delta=0.095$ at $\sim 16 \mathrm{mrad}$ [3]. Many energy-filtered DBI/H methods are now being conducted to make these long-sought-after lateral coherence measurements of the quasi-particles. An important consideration is dynamic diffraction of the energy loss electrons requiring good knowledge of the diffracting condition, scattering angle and energy loss [4]. Using ionization energy loss electrons (100s eV), a new method of magnetic microscopy using energy-filtered DBI/H may also be possible (Fig. 2) [5], which would be 
capable of measuring the magnetic potential of domains at the nanoscale, as well as, possibly the coherence of the magnon.

[1] R.A. Herring, Ultramicroscopy 104 (2005) 261.

[2] R.A. Herring, Ultramicroscopy 108 (2009) 688.

[3] R.A. Herring, J. Electron Microscopy 1-9 (2009) doi:10.1093/jmicro/dfn027.

[4] P. Schattschneider and J. Verbeeck, Ultramicroscopy 108 (2008) 407.

[5] P. Schattschneider, private communication.

Grants from UVic, NSERC, CFI and BCKDF are gratefully appreciated.

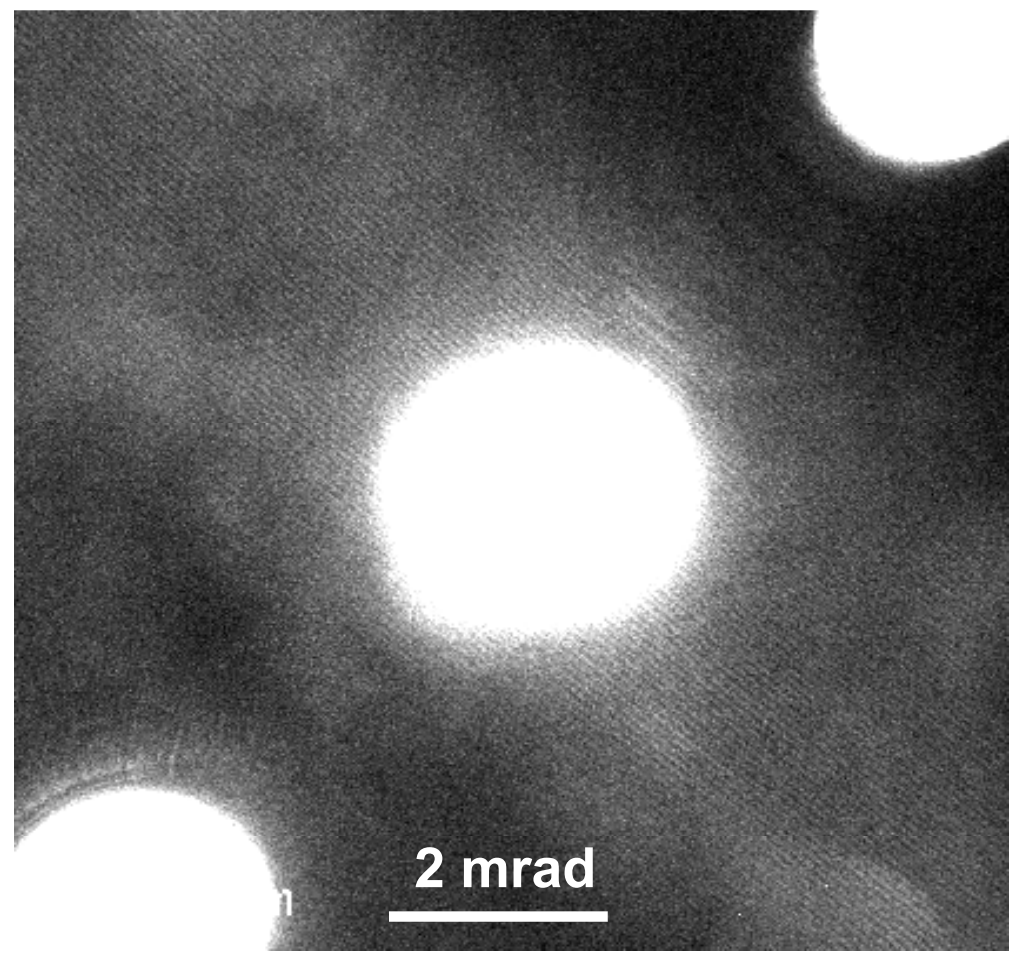

Fig. 1. An over-exposed hologram revealing the presence of fringes extending out to high scattering angles on the diffraction plane.

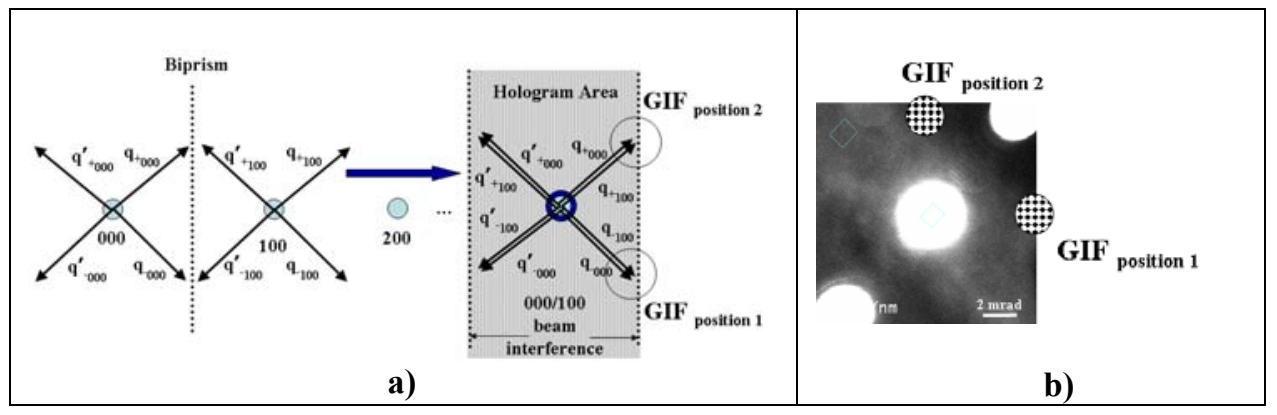

Fig. 2. a) Schematic showing the magnetic potentials, which can be determined for a domain from the intensity difference in a hologram between position 1 and position 2 . The coherence of the magnon would be determined from the high intensity central region. b) Shows placement of the intensity detector(s) on an energy-filtered DBI/H hologram at position 1 and position 2 . 